\title{
Comparison of an expert system with other clinical scores for the evaluation of severity of asthma
}

\author{
V. Gautier*, H. Rédier*, J.L. Pujol*, J. Bousquet*, H. Proudhon**, \\ C. Michel**, J.P. Daurès ${ }^{+}$, F.B. Michel*, Ph. Godard*
}

Comparison of an expert system with other clinical scores for the evaluation of severity of asthma. V. Gautier, H. Rédier, J.L. Pujol, J. Bousquet, H. Proudhon, C. Michel, J.P. Daurès, F.B. Michel, Ph. Godard. CERS Journals Ltd 1996.

ABSTRACT: "Asthmaexpert" was produced at the special request of several clinicians in order to obtain a better understanding of the medical decisions taken by clinical experts in the management of asthmatic patients. In order to assess the severity of asthma, a new score called Artificial Intelligence score (AI score), produced by Asthmaexpert, was compared with three other scores (Aas, Hargreave and Brooks).

One hundred patients were enrolled prospectively in the study during their first consultation in the out-patient clinic. Distribution of severity level according to the different scores was studied, and the reliability between AI and other scores was evaluated by Kappa and MacNemar tests. Correlations with functional parameters were performed.

The AI score assessed higher levels of severity than the other scores (Kappa=18, 28 and $10 \%$ for Aas, Hargreave and Brooks, respectively) with significant MacNemar test in all cases. There was a significant correlation between AI score and forced expiratory volume in one second (FEV1) $(r=0.73)$.

These data indicate that the AI score is a severity score which defines higher levels of severity than the chosen scores. Correlations for functional parameters are good. This score appears easy to use for the first consultation of an asthmatic patient. Eur Respir J., 1996, 9, 58-64.
*Service des Maladies Respiratoires, CJF INSERM 92, Hôpital universitaire Arnaud de Villeneuve, Montpellier, France. **Laboratoire de Biomathématiques, Faculté de Médicine, Marseille, France. +Département d'Informatique Médical, Hôpital Lapeyronie Montpellier, France.

Correspondence: Ph. Godard, Service des Maladies Respiratoires, CJF INSERM 92, Hôpital universitaire Arnaud de Villeneuve, 371 Avenue du Doyen Giraud, 34295 Montpellier Cedex 5, France.

Keywords: Asthma, expert system, scores severity

Received: December 61993

Accepted after revision August 41995

This work has been supported by a grant from Glaxo (France) and ARIA (Association pour la Recherche en Intelligence Artificielle, 9 boulevard Berthelot, 34000 Montpellier, France).
There has been a worldwide increase in asthma morbidity and mortality [1-3] as a result both of prevalence and severity of the disease $[4,5]$. These trends are worrying because they come when a better understanding of the physiopathology of asthma and when more effective and safer drugs for its management are available. Some explanations have been advanced but most of the reports point to underdiagnosis and underuse of adequate treatment [6]. The assessment of severity appears to be a milestone of good therapeutic decisions. Thus, various national groups have published guidelines achieved by consensus for the better management of asthma [7, 8]. However, definition of the severity is not standardized. Some authors consider the symptoms at a specific time [9], whilst others take into account events during precedent weeks or months $[10,11]$. Moreover, there is a lack of agreement of components which need to be considered. Thus, some scores take into account clinical symptoms, whilst others are based mainly on peak flow assessment or measurement of nonspecific bronchial hyperreactivity. Some authors grade asthma in three steps (mild, moderate and severe) [8, 12, 13], others grade in four [9, 14] or five steps [10].

Medical expert systems (ES) have great potential to help clinicians improve health-care. Some ES in pneumology have been produced and a few of these have diagnostic purposes [15-18]. Over the past few years, an ES, called Asthmaexpert, has been designed by several chest physicians. The objective was mainly to understand the medical reasoning in the management of the first visit of a new asthmatic patient and to formalize the knowledge of several experts in asthmology. After the history, clinical examination and pulmonary function test data have been compiled, the ES uses these decisionmaking rules to provide conclusions regarding the severity of the disease (scored 1-5) and precipitating factors involved. The ES also advises on the therapeutic plan to be adopted and suggests further investigations that may be required.

The aim of the present study was firstly to compare the level of severity assessed by the Artificial Intelligence (AI) score with the level of severity assessed by three severity scores described by HARGREAVE et al. [9], AAS [10] and BROOKs et al. [11]; and secondly, to study correlations with functional parameters.

\section{Material and methods}

Patients and study design

One hundred patients were prospectively enrolled in the study by the same investigator. Every adult asthmatic 
patient (more than 18 yrs of age) who visited the chest physician for the first time could be enrolled, as long as no emergency treatment was required. Asthma was defined according to the criteria of the American Thoracic Society (ATS) [19]. If the reversibility of the FEV1 after $\beta_{2}$-agonist had not been recorded within the previous 6 months or on the day of the consultation, the expert had to test it during the month following the consultation. This first consultation included systematic medical questions, physical examination, lung function tests and standard chest roentgenography. Blood eosinophils, skin prick tests, total serum immunoglobulin E (IgE) levels and specific IgE were performed only if clinically required. All patients were seen by the same practioner who scored all the patients according to criteria defined by HARGREAVE et al. [9], AAs [10] and BRoOKs et al. [11]. After compiling the data, Asthmaexpert provided a score of severity from 1 to 5 .

\section{Methods}

Paradigm of Asthmaexpert. Expert systems (ES) are computer programs that typically contain large amounts of knowledge for making decisions about specific problem domains as an area of medicine. Many expert systems are designed to duplicate a physician's reasoning and, in effect, attempt to tell the physician how to practise medicine [20-22].

Asthmaexpert was designed in order to answer the questions asked during the first consultation of an adult asthmatic patient, so long as no emergency treatment is required. Asthmaexpert has been described previously [23]. Briefly, the knowledge base was established by several expert chest physicians in regular contact with a knowledge engineer. All of the experts were French senior chest physicians, with a particular interest in asthma. They were able to formalize the knowledge through about 1,000 rules. After examining a patient, the physician enters the data on the Asthmaexpert program, which takes about $10 \mathrm{~min}$. A case report form (CRF) with the indispensable items needed to collect all the information to manage an asthmatic patient has been produced by experts. The medical concepts are represented by "NExpert objects" (Neuron Data - USA). The program has been implemented on a Macintosh II CX. The system requires 4 megabytes of memory.

Conclusions given by Asthmaexpert. After compiling the patient's data, the system proposes five different conclusions: 1) severity of asthma assessed on a five step scale (AI score); 2) the precipitating factors; 3 ) advice, such as prevention of exercise-induced asthma or methods to avoid mites in the home; 4) further investigations which might be required, e.g. dosage of specific IgE or sinus radiography; and 5) a prescription giving the family and subfamily of drugs and the dosage.

Description of score of severity. For assessment of severity, the AI score uses 64 rules and takes into account clinical features, instability of the disease, pulmonary function testing and current treatment.
The clinical features used are: frequency, intensity and timing of asthma attacks; nocturnal events within the past month and previous year; degree of dyspnoea at rest and/or after exertion; and clinical findings (cough, dyspnoea, sputum, wheezing and/or chest tightness).

Instability of the disease takes into account: nocturnal events; number of sudden severe exacerbations occurring in the last month or year with urgent visits to hospital, emergency room or doctor's surgery; and when available, peak expiratory flow rates (PEFRs) recorded for several days before the consultation. Rules have been drawn up to assess the importance of the instability (nil, mild, moderate or severe). Instability is: nil if PEFR variability is $<10 \%$; mild if PEFR variability is $10-20 \%$; moderate if PEFR variability is $20-30 \%$; and severe if PEFR variability is $>30 \%$.

Bronchial obstruction is defined by the ES according to the following parameters: 1) obstruction is absent if forced expiratory volume in one second (FEV1), forced vital capacity (FVC) and $\mathrm{FEV} 1 / \mathrm{FVC}$ are $>85 \%$ of predicted; 2) mild if FEV1 is $>70 \%$ pred with FEV1/FVC $60-85 \%$ pred; 3 ) medium if FEV1 is $50-85 \%$ pred with FEV1/FVC 40-60\% pred; 4) severe if FEV1 is $50-85 \%$ pred with $\mathrm{FEV} 1 / \mathrm{FVC}<40 \%$ pred, or if $\mathrm{FEV} 1$ is $<50 \%$, or if $\mathrm{FVC}$ is $<70 \%$.

Current treatment $\left(\beta_{2}\right.$-agonists and corticosteroid therapy) and doses of oral corticosteroid therapy used during the previous year also serve as a guide to assess severity.

The other scores. The Aas score is a five step scale clinical score which takes into account events that took place during the previous year. The parameters are: number and intensity of crises; need of institutional treatment with or without corticosteroid medication (table 1).

The Hargreave score is the result of the thoughtful workshop deliberation of many investigators interested and experienced in the assessment and treatment of asthma. Severity of asthma is judged at a particular time on the basis of symptoms, need for inhaled $\beta_{2}$-agonist and airflow rates distributed into four classes (table 2).

Brooks proposes a score named Disease Severity Score (DSS) where a numerical value is based on six parameters expressing clinical features of asthma regularly present during the previous 6 months. Each of these components of the DSS score is arbitrarily divided into six categories representing increasingly more severe features of the specific parameters. The range of scoring system for the DSS with the above system is 6-30, with a minimum score of 6 and a maximum score of 30 (table 3). To facilitate statistical analysis, we arbitrarily divided these results into five stages: Stage I 6-10; Stage II 11-15; Stage III 16-20; Stage IV 21-25; and Stage V 26-30.

\section{Statistical analysis}

The Kappa test [24] was used to compare the level of severity assessed by AI versus the other scores; and the MacNemar test [25] was used to evaluate the significance. The aim of the Kappa test was to evaluate the 
Table 1. - Aas score

1. Less than five episodes per year with less than 7 days duration of symptoms and functional restriction each time, and long symptom-free intervals with apparently normal lung function.

2. Five to 10 episodes per year with less than 7 days duration of symptoms and functional restriction each time, and long symptom-free intervals with apparently normal lung function.

3. More than 10 episodes per year with less than 7 days duration of symptoms and functional restriction each time, and long symptom-free intervals with apparently normal lung function, or more prolonged periods (totalling 12 weeks or more per year) with symptomatic bronchial obstruction or apparently impaired lung function.

4. More than five episodes per year with prolonged obstruction (totalling 6 months or more per year) following most episodes, or chronic symptomatic obstruction with restriction of function. Bronchial asthma in need of institutional treatment and/or continuous use of corticosteroid medication (any route) to classify for grade III or better.

5. Chronic, incapacitating asthma with severe, acute exacerbations in spite of continuous medication following adequate and safe dosage regimens.

(From AAs [10]).

Table 2. - Hargreave score

1. Asthma is controlled: minimal symptoms, ideally none; normal activities of daily living; inhaled $\beta$-agonist not more than twice daily; airflow rates normal or near normal at rest, airflow rates normal after inhaled $\beta$-agonist; daily variation of PEFR $<20 \%$; minimal side-effects from medication.

2. Symptoms slightly more than when asthma is controlled; inhaled $\beta$-agonist needed from one to three or four times daily; PEFR or FEV1 about $85 \%$ of predicted or of known best result; PEFR variability 20-30\%.

3. Symptoms occurring repeatedly and disturbing sleep or syptoms that are present on waking; inhaled $\beta$-agonist more often or more than four times daily; PEFR or FEV1 60-85\% of predicted or of known best result; PEFR variability $>30 \%$.

4. Symptoms at rest, not completely reversed by inhaled $\beta$-agonist; PEFR or FEV $1<60 \%$ of predicted value.

PEFR: peak expiratory flow rate; FEV1: forced expiratory volume in one second. (From HARGREAVE et al. [9]).

Table 3. - Disease Severity Score (DSS)

The six clinical parameters are:

1. Number of acute asthma attacks that required physician's treatment (including medication by injection) at office, emergency room, or hospital. Score from 1 to 5: none; at least one; two; three or four; or more than four attacks.

2. Frequency of attack of wheezing and/or chest tightness that occurred during an average day. Score from 1 to 5: none; at least one; two; three or four; five or more occurrences.

3. Frequency of attack of wheezing and/or chest tightness that occurred during an average night. Score from 1 to 5: sleep throughout the night; awakened once; awakened twice; awakened 3-4 times; or awakened by asthma almost hourly.

4. Cough score for average day or night. Score from 1 to 5: no cough; occasional cough but not seriously disturbing; quite troublesome and frequent; or distressing most of the time, day or night.

5. Degree of shortness of breath with exertion. Score from 1 to 5: can walk indefinitely; gets short of breath with strenuous exertion; shortness of breath with moderate exertion, such as climbing one or two flights of stairs; shortness of breath with minimal exertion, such as climbing one half to one flight of stairs; and shortness of breath at rest.

6. Therapy that the patient required to control asthma. Score from 1 to 5 : none; $\beta$-agonist bronchodilatators used intermittently only; oral theophylline used continuously; regular use of cromolyn, either aerosol or oral corticosteroids. The range of scoring for the DSS with the above system was 6-30, with a minimum possible score of 6 and a maximum score of 30 .

(From BROOKs et al. [11]).

reliability between the level of severity assessed by two different scores. The aim of the MacNemar test was to find out whether one of these two scores underevaluated or overevaluated the severity. Spearman rank order co-efficients of correlation were calculated in order to analyse the relationship of scores versus FEV1, and FEV1/ FVC. The $\mathrm{p}$-value equating significance was $\mathrm{p}$ less than 0.05 .

Statistical analysis was calculated on a VAX computer using BMDP software. 

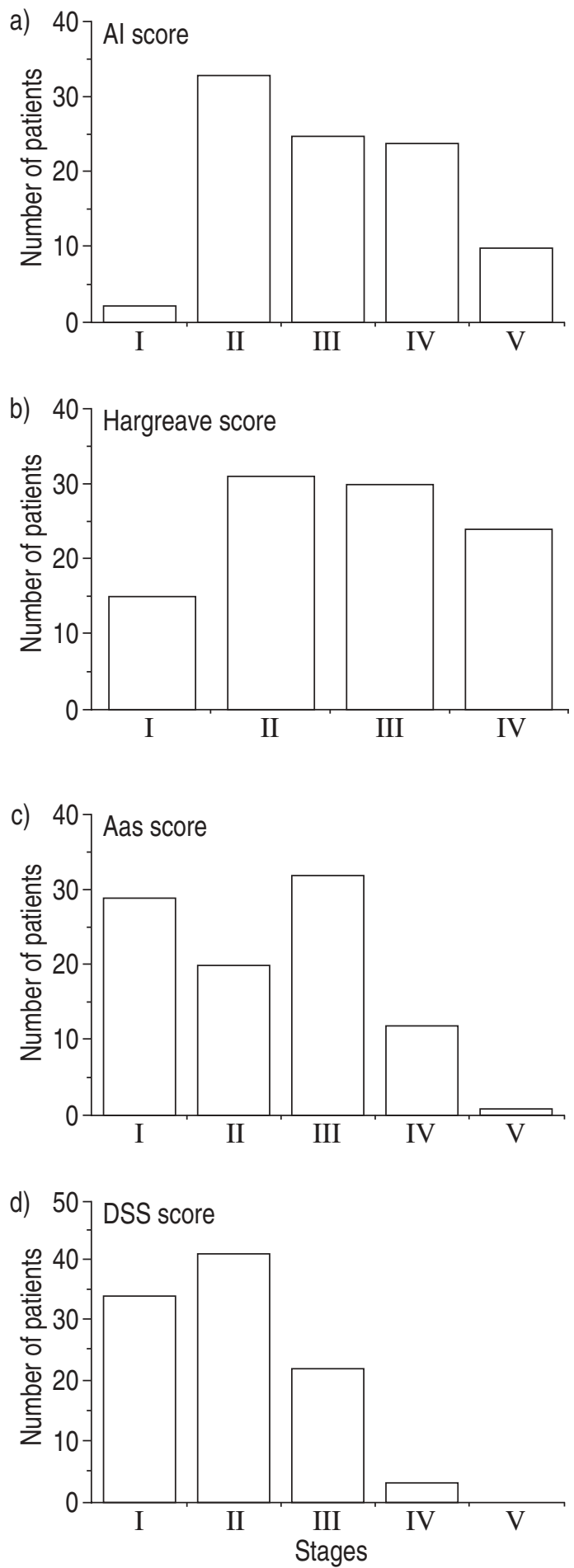

Fig. 1. - Severity classification according to the different scores.

\section{Results}

\section{Patients' characteristics}

Ninety four patients were considered for the results because in six cases ES was unable to draw a conclusion owing to a lack of information in the knowledge base. There were 46 females and 48 males aged 18-71 $\mathrm{yrs}$ (mean \pm SD, $38 \pm 15 \mathrm{yrs}$ ). The mean duration of evolution of asthma was $12 \pm 11$ (range 1-53) yrs. Concerning the
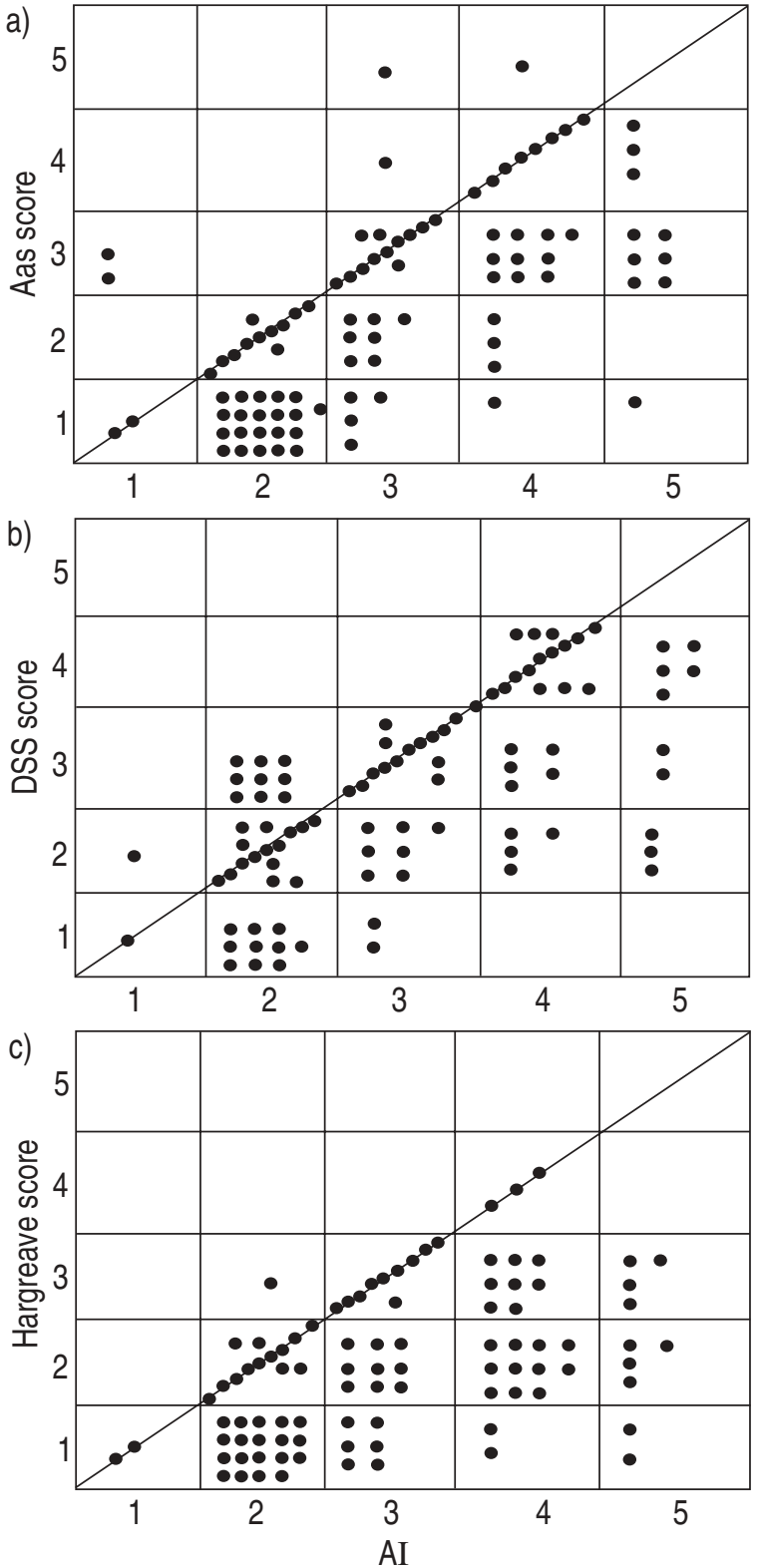

Fig. 2. - a) Test of concordance between Aas and AI score; Kappa $=18 \%$, MacNemar $\mathrm{p}<10^{-5}$. b) Test of concordance between Hargreave and AI score; Kappa $=28 \%$, MacNemar $\mathrm{p}<10^{-5}$. c) Test of concordance between DSS and AI score; Kappa $=10 \%$, MacNemar $\mathrm{p}<10^{-3}$. AI: Artificial Intelligence; DSS: Disease Severity Score.

global evolution of the previous year, 56 patients worsened, 26 were stationary, 10 had a variable evolution and 2 improved. Pulmonary function, assessed by measuring FEV1, ranged 29-129\% of predicted values, with a mean of $74 \pm 24 \%$ pred. The bronchial obstruction was judged by ES to be severe in 20 cases, medium in 22, mild in 23 and nil in 29 cases. The range of FEV1 was 29-53, 50-76, 71-91, and 87-129\% pred, in severe, medium, mild and nil obstruction, respectively.

\section{Distribution according to different scores}

The severity classification according to different scores is represented in figure 1 . There was a low level of 

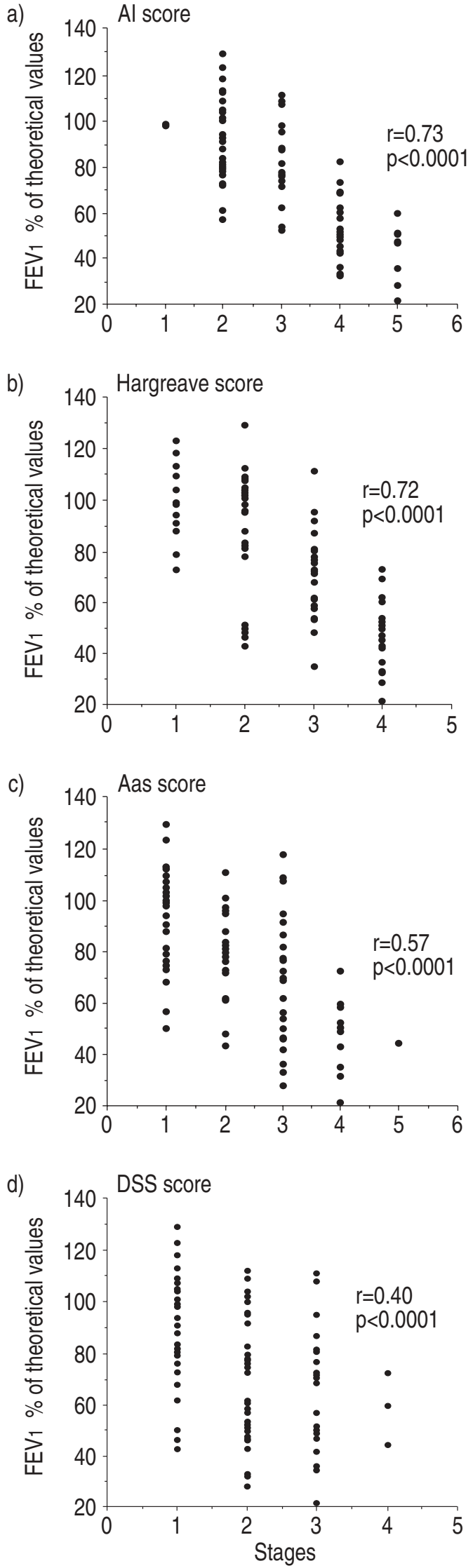

Fig. 3. - Relationship of forced expiratory volume in one second (FEV1) versus classification of asthma severity score. For definitions see legend to figure 2 . reliability between $\mathrm{AI}$ and the other scores. Kappa were 18, 28 and 10\%, for Aas, Hargreave and DSS, respectively. Fifty six patients were scored as being more severe according to the AI score than the Aas score, with a significant MacNemar test $\left(\mathrm{p}<10^{-5}\right)$ (fig. 2a). AI overevaluated 38 and 65 subjects, respectively, when compared to Hargreave and DSS (fig. 2b and c). MacNemar tests were significant for these two scores $\left(\mathrm{p}<10^{-3}\right)$.

\section{Correlations between variables}

There was a significant correlation between all the scores and FEV1. Highest correlations were observed for AI score $\left(r=0.73 ; p<0.0001 ; r^{2}=0.55\right)$ and for Hargreave $(\mathrm{r}=0.72 ; \mathrm{p}<0.0001)$ (fig. $3 \mathrm{a}$ and $\mathrm{b})$. For the Aas score and the DSS the correlations were: Aas $r=0.57$ and $\mathrm{p}<0.0001$; and DSS $\mathrm{r}=0.40$ and $\mathrm{p}<0.0001$ (fig. $3 \mathrm{c}$ and $\mathrm{d}$ ).

\section{Discussion}

In this study, we have shown that there was a relationship between the AI score and functional parameters and that the AI score tended to assess higher level of severity than the other scores.

Asthma is a complex, variable, multifactorial disorder, justifying the use of a multiparametric score as convenient to assess the severity of this disease. Correct assessment of severity of chronic asthma is essential in making a good therapeutic decision. If we consider the literature, many different scores have been published reflecting discrepancies in the definition of severity. The disagreement lies in numerous facts: the period to assess the severity; the number of steps; the parameters which need to be considered.

Almost all scores have a three step scale; however, although scores in three steps are most appropriate for teaching purposes, five steps might be useful to evaluate the various features of asthma. In previous scores, no objective measurement was used and the medical history was the most important feature to determine severity; however, we know that, if symptoms are the most sensitive indicators in some patients, other patients have a poor perception of their airflow limitation and reduction in airflow rate is more sensitive [26, 27]. On the other hand, it appears that most of the recent scores are difficult to use in daily clinical practice, since the variation of peak flow rate is not always available (in our experience only $5 \%$ of patients had measured their peak flow rate when they came to visit us for the first time), or because the assessment of bronchial hyperreactivity is either impossible or dangerous if FEV1 is too low [28, 29]. The level of previous maintenance treatment and medication requirements during previous exacerbations rarely serve as a guide in these scores. None of these scores has been validated.

These are the reasons why a group of experts in asthmology asked a knowledge engineer to build an ES. The ES has a five step scale of severity which takes into account a great number of factors, such as clinical data, functional data and current treatment. About 64 
Table 4. - Parameters to determine the severity according to the different scores

\begin{tabular}{lcccc}
\hline & AI & Aas & Hargreave & DSS \\
\hline Fc paroxystic crises & + & + & + & + \\
Intensity of crises & + & - & - & - \\
Diurnal/nocturnal & + & - & - & + \\
Severe crises & + & - & - & + \\
Dyspnoea & + & + & + & + \\
$\beta_{2}$-consumption & + & - & + & + \\
GCC.yr-1 & + & + & - & + \\
IC & + & + & - & + \\
PEFR & + & - & + & - \\
FEV1 & + & - & + & - \\
\end{tabular}

GCC $\cdot \mathrm{yr}^{-1}$ : general consumption of corticosteroids per year; IC: inhaled corticosteroids; Severe crisis: crisis requiring physician's treatment with or without hospitalization, with or without rescucitation; AI: Artificial Intelligence (score); DSS: Disease Severity Score. Fc: frequency. For further abbreviations see legend to table 2 .

rules have been designed for assessing severity using all these parameters. Although each of these parameters is normally used to assess the severity of asthma, this was the first time they had been used together in a severity score (table 4). However, Asthmaexpert is able to draw conclusions about severity even if parameters such as PEFR are not available. Asthmaexpert is easy to use during the first visit of an asthmatic patient because it avoids the inflexibility of previous classifications and provides a rapid conclusion about the level of severity; given the clinical data, ES produces a score in 8-10 $\min$.

We chose three scores among those published. Aas score was chosen because previous studies have shown correlations between this score and functional or biological parameters [30-32]. Hargreave score was used even though the authors have chosen to assess severity of asthma at one specific time. They consider that the allocation of a level of severity is based on limited information, and we wanted to determine whether the variation in numbers of months evalulated could explain discrepancies in assessement of severity. The DSS was chosen because each of the parameters was very accurate. These scores do not look at the same time-frame, the parameters included are different, but, even though they were not developed for the same purpose, the authors have concluded that their scoring systems may be applicable to practising physicians.

The system provided conclusions for 94 patients; the absence of conclusions in six patients was the consequence of a lack of information for the knowledge base. Asthmaexpert's knowledge base is certainly incomplete, but it is being kept up to date. According to data in the literature, we found no correlation between severity and age or duration [21], whatever the scores used. The analysis severity scores and FEV1 showed a good level of correlation for AI and Hargreave scores. These results underlined the fact that AI and Hargreave integrate the FEV1 into the assessment of severity, but that FEV1 cannot be used alone to evaluate severity of asthma.
Indeed, if $\mathrm{r}^{2}$ equals 0.55 , this means that $55 \%$ of the variability of the severity score is explained by the variability of FEV1. On the other hand, $45 \%$ of the variability of the severity score is not explained by the variability of the FEV1. Thus, despite the close correlation between the AI score and FEV1, the study of the bronchial obstruction level emphasized that the value of FEV1 was not a key factor in the severity assessment for the ES.

For the distribution analysis of patients according to different scores of severity, the Kappa and MacNemar test showed that AI assessed a higher level of severity than the other scores; perhaps, because AI score takes into account all the parameters usually used to assess severity. The major consequence was that the therapeutic level was higher than that proposed by the other scores. Moreover, when there was discordance about level of severity, CRF were carefully studied by the experts to explain the difference. Thus, Asthmaexpert became a tool for permanent consensus between the experts.

Asthmaexpert is an evolving computer system that uses an extensive knowledge base. It originates from the specific request of several expert chest physicians who wanted to formalize knowledge in asthmology in order to create a dynamic consensus movement in the approach to a complex and variable illness. The tool exists, and the program has been implemented on a MacIntosh computer. The conclusions are relevant and the system has been validated $[23,33]$. The formalization of this knowledge has made it possible to obtain a first level of consensus. In order to validate the long range conclusions of the system, it would be interesting to make a prospective scoring study to determine the severity at each consultation. Although Asthmaexpert can be improved both in its performance and in its knowledge base, we believe that the system might contribute to improvement in the management of asthmatic patients. Asthmaexpert may be helpful to the general practioner and to students. Moreover, this ES may harmonize the study of asthmatic patients.

Acknowledgement: The authors wish to thank J. Baïssus for help in preparing the manuscript.

\section{References}

1. Benatar SR. Fatal asthma. N Engl J Med 1986; 314: 423-429.

2. Evans R III, Mullally DI, Wilson RW, et al. National trends in the morbidity and mortality of asthma in the US, prevalence, hospitalization and death from asthma over two decades, 1965-1984. Chest 1987; 91: 65S-74S.

3. Sly RM. Mortality from asthma, 1979-1984. J Allergy Clin Immunol 1988; 82: 705-717.

4. Gergen PJ, Weiss KB. The increasing problem of asthma in the United States. Am Rev Respir Dis 1992; 146: 823-824.

5. National Heart, Lung and Blood Institute Data Fact Sheet. Asthma Statistics. Bethesda, Md, NHLBI Education Programs Information Center.

6. Buist SA, Vollmer WM. Reflections on the rise in asthma morbidity and mortality. J Am Med Assoc 1990; 13: $1719-1720$ 
7. British Thoracic Society. Guidelines for the management of asthma. Thorax 1993; 48S: 1-24.

8. National Heart, Lung, and Blood Institute. National Institutes of Health. Bethesda, International Consensus Report on Diagnosis and Treatment of Asthma, June 1992.

9. Hargreave FE, Dolovich J, Newhouse MT. The assessment and treatment of asthma: a conference report. $J$ Allergy Clin Immunol 1990; 85: 1098-1111.

10. Aas K. Heterogeneity of bronchial asthma. Allergy 1981; 36: 3-14.

11. Brooks SM, Bernstein L, Raghuprasad PK, Maccia CA, Mieczkowski L. Assessment of airway hyperresponsiveness in chronic stable asthma. J Allergy Clin Immunol 1990; 85: 17-26.

12. Japanese Society of Allergology. Standardized criteria for evaluating the severity of adult asthma. Jap J Allergol 1980; 30: 1187-1199.

13. Richards JM, Bailey WC, Windsor RA, Martin B, Soong S. Some simple scales for use in asthma research. $J$ Asthma 1988; 25: 363-371.

14. Woolcock AJ, Jenkins CR. Assessment of bronchial responsiveness as a guide to prognosis and therapy in asthma. Med Clin North Am 1990; 74: 753-765.

15. Cummings SR, Lillington GA, Richard RJ. Estimating the probability of malignancy in solitary nodules. Am Rev Respir Dis 1986; 134: 449-452.

16. Edwards FH, Schaefer PS, Callahan S, Graeber GM, Albus RA. Bayesian statistical theory in preoperative diagnosis of pulmonary lesions. Chest 1987; 92: 888-891.

17. Edwards FH, Schaefer PS, Cohen AJ, et al. Use of artificial intelligence for the preoperative diagnosis of pulmonary lesions. Ann Thorac Surg 1989; 48: 556559.

18. Kunz JC, Fallot RJ, McClung DH. A physiological rulebased system for interpreting pulmonary function test results. Proc Comp Crit Care Pulm Med 1979; 242: 1279-1282.

19. Standard for the diagnosis and care of patients with chronic obstructive disease (COPD) and asthma. ATS statement. Am Rev Respir Dis 1987; 136: 225-245.

20. Miller R, Pople H, Myers J. Internist-I, an experimental computer-based diagnostic consultant for general internal medicine. N Engl J Med 1982; 307: 468-476.

21. Shortliffe EH. Computer-based medical consultations: MYCIN. New York, American Elsevier, 1976.

22. Miller PL. Expert critiquing anesthesic management: the "Attending" computer system. Anesthesiology 1983; 58: 362-369.

23. Rédier H, Daures JP, Michel C, et al. Assessment of the severity of asthma by an expert system: description and evaluation. Am J Respir Crit Care Med 1995; 151 : 345-352.

24. Snedecor GW, Cochran WG. In: Statistical methods. 5th edn. Ames, Iowa State University Press, 1956; pp. 199-201.

25. Armitage P. In: Statistical Methods in Medical Research. Oxford, Blackwell Scientific Publication, 1971; p. 123.

26. Warner JO, Götz M, Landau I, et al. Management of asthma: a consensus statement. Arch Dis Child 1989; 64: 1065-1079.

27. Dhand R, Kabra S, Malik SK. Use of a visual analogue scale for assessment of the severity of asthma. Respiration 1988; 54: 255-262.

28. Rubinfeld AR, Pain MCF. Relationship between bronchial reactivity, airway caliber and severity of asthma. Am Rev Respir Dis 1977; 115: 381-387.

29. Eiser NM, Kerrebijn KF, Quanjer PH. Guidelines for standardization of bronchial challenges with (nonspecific) bronchoconstricting agents. Bull Eur Physiopathol Respir 1983; 19: 495-514.

30. Bousquet J, Chanez P, Lacoste JY, et al. Eosinophilic inflammation in asthma. $N$ Engl J Med 1990; 323: 1033-1039.

31. Cluzel M, Damon M, Chanez P, et al. Enhanced alveolar cell luminol-dependent chemiluminecence in asthma. J Allergy Clin Immunol 1987; 80: 195-201.

32. Bousquet J, Chanez P, Lacoste JY, et al. Indirect evidence of bronchial inflammation assessed by titration of inflammatory mediators in BAL fluid of asthmatic patients. J Allergy Clin Immunol 1991; 88: 649-660.

33. Ray P, Vervloet D, Charpin D, et al. Evaluation of atopy by an expert system: description of the data base. Clin Exp Allergy, (in press). 